\title{
Atrial Activity Extraction for Atrial Fibrillation Analysis Using Blind Source Separation
}

\author{
José Joaquín Rieta*, Francisco Castells, César Sánchez, Vicente Zarzoso, Associate Member, IEEE, and José Millet
}

\begin{abstract}
This contribution addresses the extraction of atrial activity (AA) from real electrocardiogram (ECG) recordings of atrial fibrillation (AF). We show the appropriateness of independent component analysis (ICA) to tackle this biomedical challenge when regarded as a blind source separation (BSS) problem. ICA is a statistical tool able to reconstruct the unobservable independent sources of bioelectric activity which generate, through instantaneous linear mixing, a measurable set of signals. The three key hypothesis that make ICA applicable in the present scenario are discussed and validated: 1) AA and ventricular activity (VA) are generated by sources of independent bioelectric activity; 2) AA and VA present non-Gaussian distributions; and 3) the generation of the surface ECG potentials from the cardioelectric sources can be regarded as a narrow-band linear propagation process. To empirically endorse these claims, an ICA algorithm is applied to recordings from seven patients with persistent AF. We demonstrate that the AA source can be identified using a kurtosis-based reordering of the separated signals followed by spectral analysis of the sub-Gaussian sources. In contrast to traditional methods, the proposed BSS-based approach is able to obtain a unified AA signal by exploiting the atrial information present in every ECG lead, which results in an increased robustness with respect to electrode selection and placement.
\end{abstract}

Index Terms-Atrial fibrillation, blind source separation, ECG, forward electrocardiography problem, independent component analysis, QRS cancellation.

\section{INTRODUCTION}

A TRIAL fibrillation (AF) is the most common sustained arrhythmia encountered by clinicians and occurs in approximately $0.4 \%-1.0 \%$ of the general population. Its prevalence increases with age, and up to $10 \%$ of the population older than 80 years has been diagnosed with AF. With the projected growth of the elderly population, the prevalence of AF will certainly increase [1]. There is also increasing awareness that $\mathrm{AF}$ is a major cause of embolic events which in $75 \%$ of cases are complicated by cerebrovascular accidents [2], [3]. AF is often asso-

Manuscript received February 21, 2003; revised July 28, 2003. This work was supported in part by TIC2002-00957 and the research incentive program of the Polytechnic University of Valencia. Asterisk indicates corresponding author.

*J. J. Rieta is with the Bioengineering Electronic and Telemedicine Research Group, Electronic Engineering Department, Polytechnic University of Valencia. EPSG, Carretera Nazaret Oliva s/n, 46730, Gandía, Valencia, Spain (e-mail: jjrieta@eln.upv.es).

F. Castells and J. Millet are with the Bioengineering Electronic and Telemedicine Research Group, Electronic Engineering Department, Polytechnic University of Valencia. EPSG, Carretera Nazaret Oliva s/n, 46730, Gandía, Valencia, Spain.

C. Sánchez is with the Bioengineering Electronic and Telemedicine Research Group, University of Castilla-La Mancha, 16071 Cuenca, Spain.

V. Zarzoso is with the Signal Processing and Communications Group, Department of Electrical Engineering and Electronics, The University of Liverpool, L69 3GJ Liverpool, U.K.

Digital Object Identifier 10.1109/TBME.2004.827272 ciated with heart disease but a significant proportion of patients (about 30\%) have no detectable heart disease. Symptoms such as occasionally disabling haemo-dynamic impairment and a decrease in life expectancy are among the untoward effects of atrial fibrillation, resulting in an important morbidity, mortality, and an increased cost for the health care provider [1], [2]. In this respect, $\mathrm{AF}$ has been the subject of arousing interest and intensive clinical research in recent years.

The proper analysis and characterization of AF from electrocardiogram (ECG) recordings-regardless of the leads considered-requires the extraction or cancellation of the signal components associated with ventricular activity (VA), that is, the QRS complex and the T-wave (QRS-T). Unfortunately, a number of facts hinder this operation. First, atrial activity (AA) presents in the ECG much lower amplitude - in some cases well under the noise level- than its ventricular counterpart. Additionally, both phenomena possess spectral distributions that notably overlap, rendering linear filtering solutions unsuccessful.

Methods reported in the literature to cancel out VA in the ECG involve direct suppression of the QRS complex and T-wave through the subtraction of a fixed template [4]-[6]. Also, the use of an adaptive template in conjunction with the correct spatiotemporal alignment of every QRS complex has proven to be very effective [7]. All of these methods-though different in their performance-share similar limitations such as high sensitivity to QRS morphological changes in [4]-[6] and their inability to eliminate artifacts from electrode movement or ectopic beats in [4]-[7]. More recent methods have focused on extracting the VA using artificial neural networks and subtracting it from the ECG [8] or on the decomposition of the original ECG in a set of coefficients that obtain the AA using discrete packet wavelet transform [9]. A common limitation of all of the previously mentioned methods is their inability to exploit the spatial diversity of an ECG recording.

However, the key observation that AA and VA are decoupled [1], [6] introduces a new interesting perspective which does not rely on direct QRS-T elimination. Under certain assumptions, the AA extraction problem accepts a formulation based on blind source separation (BSS) of instantaneous linear mixtures [10], in which atrial and ventricular source contributions to be appear mixed at the electrode outputs in the ECG. Hence, the separation of AA sources through a suitable BSS method would allow the reconstruction of atrial contribution to each electrode free from VA and other disturbances. The multichannel signal processing standpoint adopted in the BSS approach aims at an effective utilization of the atrial information present in all ECG leads. Two main families of BSS techniques for AA extraction have been proposed, based on principal component analysis (PCA) [11], 
[12] and independent component analysis (ICA) [13], [14], respectively. PCA methods search for a solution, using secondorder statistics (SOSs), that decorrelates the input signals. By contrast, the assumptions that AA and VA are independent at orders higher than two and do not present random Gaussian distributions [6], [7] may be exploited to separate AA from VA by imposing the necessary higher order statistical conditions. If such assumptions are fulfilled, the application of ICA-based methods makes it possible to reconstruct the atrial contribution to each electrode free from VA and other large-amplitude nuisance signals, like respiration artifacts, ectopic beats, or muscular noise.

One of the most important research areas where ICA techniques have proven their success is precisely in biomedical engineering. Today the use of BSS is well known in electroencephalogram and magnetoencephalogram applications [15], [16] or in the extraction of the fetal ECG from maternal cutaneous recordings [17]. Regarding the ECG, examples of the application of BSS-based methods are the separation of breathing artifacts, muscular noise, and other disturbances [18], [19], analysis of ST segments for ischemia detection [20], identification of humans using the ECG [21], and ventricular arrhythmia detection and classification [22].

In the present contribution, a new application of BSS to the multilead ECG is presented. We show the suitability of ICA techniques to extract the AA present in the ECG of patients with persistent $\mathrm{AF}$ episodes. It is argued that $\mathrm{AA}$ and $\mathrm{VA}$ are generated by independent sources of bioelectric activity, that this activity exhibits non-Gaussian character, and that the ECG recordings fulfil the instantaneous linear model. To empirically validate these claims, an ICA method is applied to real recordings obtained from patients suffering from AF. A simple yet effective method for the identification of AA from the estimated sources is put forward, based on higher order statistics (HOS) (more specifically, the fourth-order marginal cumulant or kurtosis) and spectral analysis [14], [23].

The paper is structured as follows. Section II justifies the assumptions that lead to the suitability of the ICA-based BSS approach to the AA extraction problem. Section III develops a method for the identification and reconstruction of AA from the separated sources of cardioelectric activity. Section IV summarizes the results obtained from the application of the ICAbased AA extraction technique on real multilead ECG signals recorded from AF patients, and the results are discussed in Section V. Section VI presents the concluding remarks.

\section{AF BSS MODEL}

If BSS methods based on ICA are to be applied to the AA extraction from the 12-lead ECG, the fulfillment of three basic considerations regarding AA, VA and the fashion in which both activities arise on the body surface must first be justified: independence of the sources, non-Gaussianity, and observations generated by instantaneous linear mixing of the bioelectric sources [24]. This section begins with an outline of the basic mathematical principles behind the BSS of instantaneous linear mixtures. Then, physical mechanisms of AF generation give strong support to the independence and non-Gaussianity of AA and VA. Next, the validity of the instantaneous linear mixing model for the ECG is endorsed through the matrix solution for the forward problem of electrocardiography. The corroboration of these conditions make it possible to assume that the ECG of a patient in AF satisfies the BSS instantaneous linear mixture model, thus justifying the application of ICA.

\section{A. BSS Principles}

The BSS consists of recovering a set of source signals from the observation of linear mixtures of the sources [10], [25]. The term "blind" emphasizes that nothing is known about the source signals or the mixing structure, the only hypothesis being the source mutual independence [24]. Mathematically, let us denote by $\mathbf{s}(t)=\left[s_{1}(t), s_{2}(t), \ldots, s_{N}(t)\right]^{T} \in \Re^{N}\left({ }^{T}\right.$ stands for the transpose operator) the vector that represents the $N$ source signals and $\mathbf{x}(t)=\left[x_{1}(t), x_{2}(t), \ldots, x_{M}(t)\right]^{T} \in \Re^{M}$ the $M$ sensor output vector, i.e., the observation vector. It is assumed that $M \geq N$, so that there are at least as many sensors as sources. In the noiseless case, the BSS model for instantaneous linear mixtures reads

$$
\mathbf{x}(t)=\mathbf{A s}(t)
$$

where $\mathbf{A} \in \Re^{M \times N}$ is the unknown mixing matrix. The objective of BSS is to estimate $\mathbf{s}(t)$ and $\mathbf{A}$ from the exclusive knowledge of $\mathbf{x}(t)$. To achieve the source separation, a linear transformation $\mathbf{W} \in \Re^{N \times M}$ is sought such that the components of the output signal vector $\mathbf{x}(t)$ become statistically independent, thus representing an estimate of the sources

$$
\hat{\mathbf{s}}(t)=\mathbf{W x}(t)
$$

except for (perhaps) scaling and permutation, which are considered admissible indeterminacies.

Some authors have proposed the use of PCA to solve the model of (1) [11]. However, it is important to remark that the success of PCA relies heavily on the orthogonality of the columns of the mixing matrix. However, in general, there is no reason why bioelectrical sources of the heart should be spatially orthogonal to one another in the ECG. This orthogonality condition can only be forced through appropriate electrode placement, as was previously emphasized in the context of the fetal ECG extraction problem [26], [27] and the cancellation of artifacts in the electroencephalogram [16]. As a consequence, PCA is not expected to separate each source from the ECG with a quality similar to that of ICA. Moreover, PCA methods assume sources with a Gaussian distribution, which is not the case for AA and VA in the AF problem (as will be justified in Section II-C). In general, the measurement of independence for non-Gaussian signals can be carried out more accurately using HOS, rather than SOS, like PCA methods do.

By contrast, ICA does not introduce any restriction on the geometrical structure of the mixing matrix (apart from the linear independence of its columns) and, in addition, takes into account the non-Gaussian nature of the source signals. Consequently, ICA arises as a more sensible approach to this problem.

Several ICA techniques have been proposed mainly based on HOS and information theory [28], due to their ability to measure statistical independence. In practice, additive measurement 
noise and other disturbances (e.g., mains interference) are unavoidably present in the sensor outputs of (1). It is usually a very plausible assumption to consider the noise signals independent of the bioelectric sources of interest. When the number of electrodes is larger than the number of bioelectric sources, certain degrees of freedom are available for part of the additive noise to be extracted as separate source signals (as will be observed in the experimental results of Section IV). However, in the general case, the effective treatment of noisy observations in BSS [28], as well as in other signal processing problems, remains an open issue, which is beyond the scope of this paper.

\section{B. Mechanisms of $A F$}

One normal cardiac cycle is started at the sinus node with the depolarization of the right atrium and spreads toward the entire atria in a well-ordered manner. Atrial depolarization defines the P-wave in the ECG. Next, the depolarization impulse reaches the ventricles and their fast contraction produces the QRS complex of the ECG. Finally, ventricular repolarization produces the $\mathrm{T}$-wave and concludes the cardiac cycle [29]. The manifestation of AF, a supraventricular arrhythmia, is characterized by uncoordinated atrial activation with consequent deterioration of atrial mechanical function. AF occurs when the electrical impulses in the atria degenerate from their usual organized pattern into a rapid chaotic pattern. This disruption results in an irregular and often rapid heartbeat that is classically described as "irregularly irregular" and is due to the unpredictable conduction of these disordered impulses across the atrioventricular node [1].

On the ECG, AF is described by the replacement of consistent $\mathrm{P}$-waves by rapid oscillations or fibrillatory waves that vary in size, shape, and timing, associated with an irregular, frequently rapid ventricular response. Theories of the AF mechanism involve two processes [1]: enhanced automaticity in one or several foci [see Fig. 1(a)] and reentry involving one or more circuits [Fig. 1(b)].

The focal origin of AF is supported by experimental models and appears to be more important in patients with paroxysmal AF than in those with persistent AF. Nevertheless, the most widely accepted theory of persistent $\mathrm{AF}$ mechanisms was proposed by Moe in [30]. He postulated that AF perpetuation is based on the continuous propagation of multiple wavelets wandering throughout the atria. The fractionation of the wavefronts as they propagate results in self-perpetuating independent wavelets [2]. The number of simultaneous wavelets depends on the refractory period, mass, and conduction velocity along the atria, because these parameters present severe inhomogeneities in AF [1]. Therefore, during AF, several independent atrial propagation circuits are involved and the length of the path through which the depolarization wavefronts can travel is influenced by conduction velocity, anisotropies related to the orientation of atrial fibers and refractoriness, producing wave collision and reentry [1], [3]. Moreover, the self-perpetuating propensity of AF is justified by the electrophysiological remodeling, a phenomenon consisting in the progressive shortening of effective refractory periods, thus increasing the number of simultaneous wavelets and, as a consequence, the episode duration [1]. Through the mapping of experimentally induced AF in canine hearts, the multiple wavelet hypothesis has been

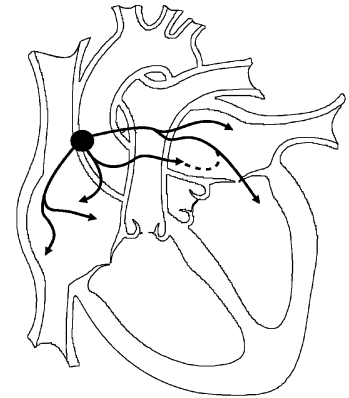

(a)

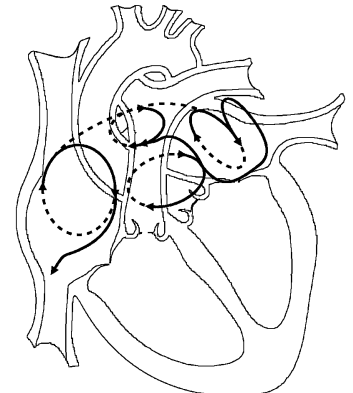

(b)
Fig. 1. Main electrophysiological mechanisms of AF. (a) Focal activation: there is an initiating focus and the resulting wavelets represent fibrillatory conduction. (b) Multiple-wavelet reentry: wavelets, indicated by arrows, randomly reenter tissue previously activated by them or by another wavelet.

proved. Similar observations have been reported in humans [1]-[3].

\section{Independence and Non-Gaussianity of AA and VA}

During an AF episode several independent wavefronts propagate simultaneously throughout the atria but only a reduced part of them will reach the AV node. Moreover, several properties of the AV node tend to limit strongly the ventricular activation. First, the excitability of cells within the AV node is significantly less than the atrial myocardium, thus meaning that the refractory period is considerably larger than in the atria [1]. Second, the AV node demonstrates decremental conduction properties; that is, the amplitude and rate of rise of cardiac action potentials decrease progressively from cell to cell. Because of this property, impulses may traverse only a portion of the AV node before blocking [2]. One clinical manifestation of this property is the phenomenon of concealed conduction, in which a atrial impulse that itself does not conduct to the ventricles may impair conduction of subsequent impulses, blocking the propagation of other impulses that otherwise would have conducted [2]. As a consequence of the aforementioned AV node properties, most of the atrial wavefronts do not reach conduction and are not able to produce ventricular depolarization.

On the other hand, the physical origin of the atrial wavefront that has been able to produce ventricular depolarization could be very variable. This uncoordinated operation of AA and VA during an AF episode makes it reasonable to regard both activities as physically independent and, in turn, as generated by statistically independent sources of cardioelectric activity. The validity of the atrio-ventricular statistical-independence assumption is in line with the findings reported by other authors in the field [1], [2], [7].

With respect to non-Gaussianity, VA presents high values within the heart beat (QRS complex) and low values in the rest of the cardiac cycle. Hence, the histogram analysis of VA reveals an impulsive, i.e., super-Gaussian, behavior [6] with typical kurtosis values above 15. On the other hand, AA of an AF episode has been accurately modeled as a sawtooth signal consisting of a sinusoid with several harmonics [7], which behaves, statistically speaking, as a sub-Gaussian random process. Moreover, when a QRS complex and T-wave cancellation algorithm, like those described in [4]-[7], is employed to cancel out VA 
over one ECG lead, it can be observed that the remaining ECG, mainly the AA, presents a sub-Gaussian behavior with negative kurtosis values. The non-Gaussian assumption of AA and VA is hence justified and will be shown in the results in Section IV.

\section{ECG Instantaneous Linear Mixture Model}

Electrocardiography involves interpretation of the potentials recorded at the body surface due to electrical activity of the heart. To this end, we use the concept of an electrical representation of the heart's activity: an equivalent source, in conjunction with a specified volume conductor to model the torso [29].

There are several physical models to represent both the cardiac current sources and the enclosing torso shape and conductivity. Source models range from simple current dipoles to complex current surfaces. Torso shape and conductivity models range from infinite homogeneous conductors to finite element models. The combination of torso and source models to calculate the body surface potentials is known as the forward problem [31]. One of the most accepted solutions for the forward problem relys on the calculation, using surface methods, of the outer body surface potentials from the epicardial (external surface of the heart) surface potentials [32]. Surface methods are based on integral equations for the potential derived by applying Green's second identity in a torso model comprising the body surface and the heart surface [33]. The general approach for finding solutions to this kind of integral equations is to discretize the problem and write one equation for each of a number of points on both surfaces and solve these equations simultaneously [31]. For $N_{B}$ points defined on the body surface, representing the field points (leads), and $N_{H}$ on the epicardium representing the source positions, it is possible to write the following set of discretized expressions as the observation point sweeps all the body and the heart surface:

$$
\begin{aligned}
& \sum_{j=1}^{N_{B}} p_{B B}^{i j} \Phi_{B}^{j}+\sum_{j=1}^{N_{H}} p_{B H}^{i j} \Phi_{H}^{j}+\sum_{j=1}^{N_{H}} g_{B H}^{i j} \Gamma_{H}^{j}=0 \\
& \sum_{j=1}^{N_{B}} p_{H B}^{i j} \Phi_{B}^{j}+\sum_{j=1}^{N_{H}} p_{H H}^{i j} \Phi_{H}^{j}+\sum_{j=1}^{N_{H}} g_{H H}^{i j} \Gamma_{H}^{j}=0
\end{aligned}
$$

where $\Gamma_{H}^{j}$ is the normal component of the potential gradient for point $j$ on the heart surface. In general, the $g_{P Q}^{i j}$ term links the potential at observation point $i$ on surface $P$ to the value of the potential gradient $\Gamma_{H}^{j}$ at point $j$ on surface $Q$, while $p_{P Q}^{i j}$ is the geometrical coefficient which weights the contribution in the observation point $i$ on surface $P$ of the potential at node $j$ on surface $Q$. Therefore, the equations can be separated into the product of a potential $\left(\Phi_{B}^{j}\right.$ or $\left.\Phi_{H}^{j}\right)$ or the gradient of a potential $\left(\Gamma_{H}^{j}\right)$ at a specific point $j$ on either one of the surfaces and a second factor (the terms with general form $p_{P Q}^{i j}$ and $g_{P Q}^{i j}$ ) based entirely on the geometry of the torso and the heart. $\Phi_{B}^{j}$ and $\Phi_{H}^{j}$ are the potential at node $j$ on the body and heart surfaces, respectively. Now expressing the summations in matrix form, we have

$$
\begin{aligned}
& \mathbf{P}_{B B} \boldsymbol{\Phi}_{B}+\mathbf{P}_{B H} \boldsymbol{\Phi}_{H}+\mathbf{G}_{B H} \boldsymbol{\Gamma}_{H}=0 \\
& \mathbf{P}_{H B} \boldsymbol{\Phi}_{B}+\mathbf{P}_{H H} \boldsymbol{\Phi}_{H}+\mathbf{G}_{H H} \boldsymbol{\Gamma}_{H}=0
\end{aligned}
$$

where $\boldsymbol{\Phi}_{B}$ and $\boldsymbol{\Phi}_{H}$ are $N_{B}$ and $N_{H}$ potential column vectors, $\boldsymbol{\Gamma}_{H}$ is a column vector of $N_{H}$ epicardial potential gradients, and the various $\mathbf{P}$ and $\mathbf{G}$ coefficient matrices are determined solely by integrations involving the geometry of the epicardial and body surfaces. Here again, the first subscript of $\mathbf{P}$ (or $\mathbf{G}$ ) represents the surface containing the observation points, having as much rows as points $\left(N_{H}\right.$ or $\left.N_{B}\right)$, and the second one represents the surface (heart or body) of integration with the number of columns equal to the number of points where the integration is computed $\left(N_{H}\right.$ or $\left.N_{B}\right)$. Solving (6) for the matrix of epicardial potential gradients $\Gamma_{H}$ and substituting the result into (5) yields

$$
\boldsymbol{\Phi}_{\mathbf{B}}=\mathbf{T}_{B H} \boldsymbol{\Phi}_{H}
$$

with $\mathbf{T}_{B H}$ defined as

$$
\begin{aligned}
\mathbf{T}_{B H}=\left(\mathbf{P}_{B B}-\mathbf{G}_{B H}\left(\mathbf{G}_{H H}\right)^{-1} \mathbf{P}_{H B}\right)^{-1} \\
\cdot\left(\mathbf{G}_{B H}\left(\mathbf{G}_{H H}\right)^{-1} \mathbf{P}_{H H}-\mathbf{P}_{B H}\right) .
\end{aligned}
$$

Equations (7) and (8) define the solution to the forward problem. The elements of matrix $\mathbf{T}_{B H}$ are the transfer coefficients relating the potential at a particular point on the epicardial surface to that at a particular point on the body surface, and they depend solely on the geometry of the epicardial and body surfaces and the conductivity of the torso.

Equation (7) shows that the electric potential in one point of the body surface can be obtained by adding the partial contributions of the heart potentials, weighted by a transfer coefficient. Obviously, (7) corresponds to a linear mixing model where a set of observations are obtained by linearly combining a set of sources. In our case, the sources are the set of bioelectric potentials in the epicardium and the observations the set of body-surface potentials.

The transfer (or mixing) matrix of (8) models the conductivity of the human torso and, in a first approximation, may be considered as an isotropic homogeneous volume conductor. A more realistic modeling of the torso can consider inhomogeneities of the volume conductor and the presence of different tissues. One can take such inhomogeneities into account by approximating the volume conductor by a collection of regions, each one of which is homogeneous, resistive, and isotropic but, at the same time retaining the results of (7) [32]. Hence, inhomogeneities and anisotropies in the human torso only modify the transfer coefficients, i.e., the elements of $\mathbf{T}_{B H}$, but do not affect the fulfillment of the model [34].

Finally, in the description of the volume conductor constituted by the human body, the capacitive component of tissue impedance is negligible in the frequency band of internal bioelectric events $(0-100 \mathrm{~Hz})$. Hence, the volume conductor currents generated by the heart's bioelectrical activity are essentially conduction currents and require only specification of the tissue resistivity. The electromagnetic propagation effect can also be neglected [29]. As a reinforcement of this assumption, the finite-difference method for solving the forward problem represents the torso geometry by a three-dimensional (3-D) grid of discrete points interconnected using resistive elements [35]. These considerations imply that time-varying bioelectric currents and voltages in the human body can be examined with the 


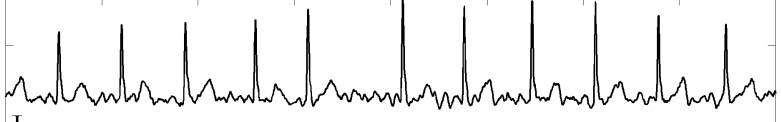
I

II

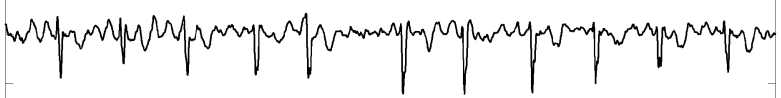

III

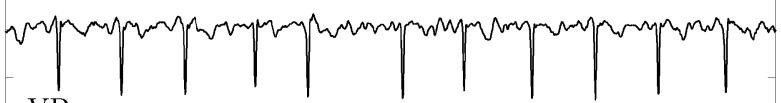

aVR

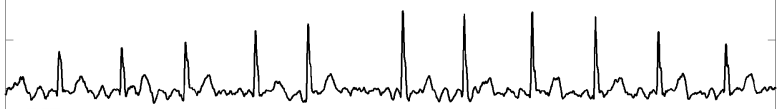
$\mathrm{aVL}$

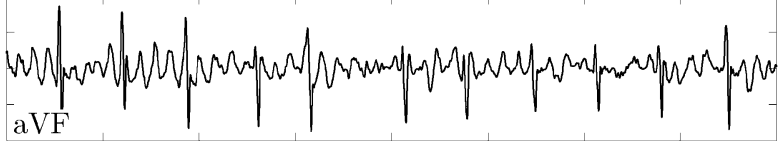

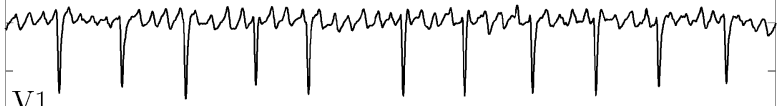

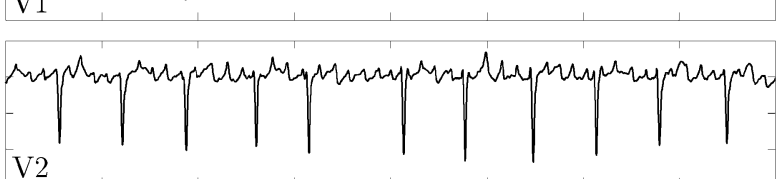
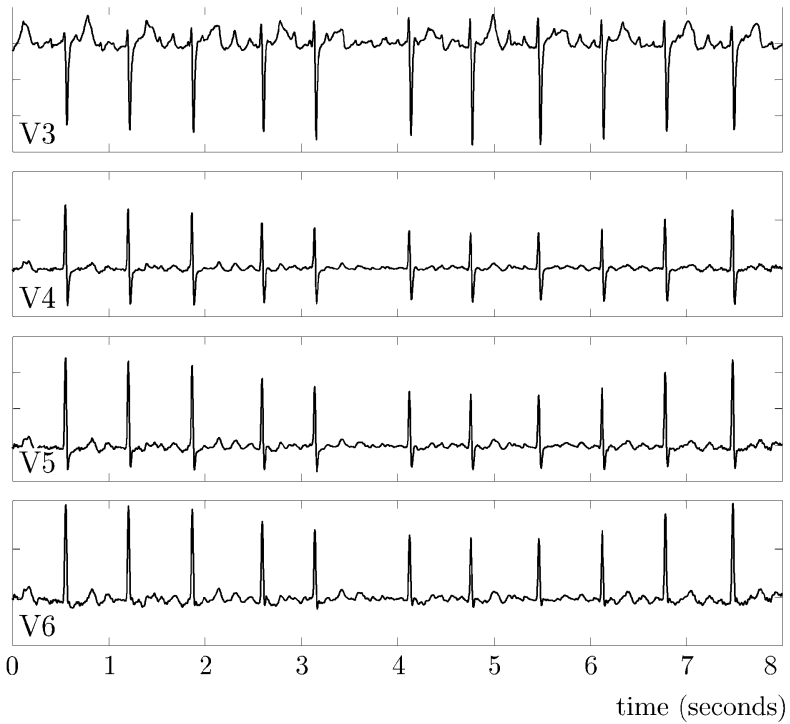

(a)

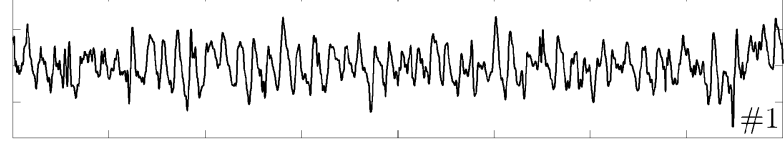

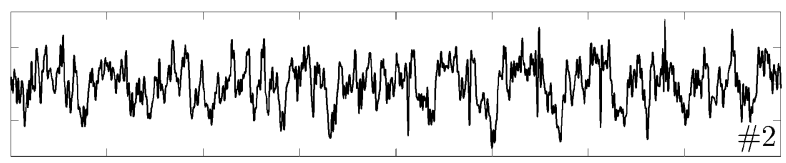

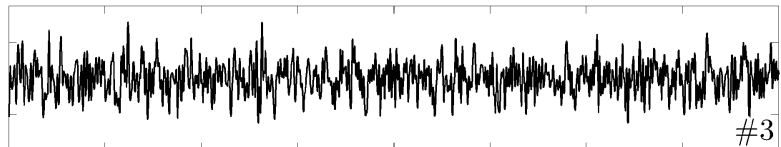

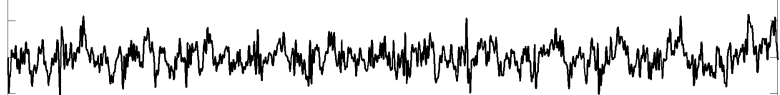

\#4

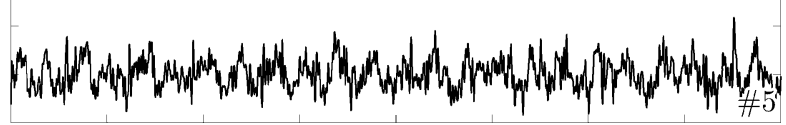

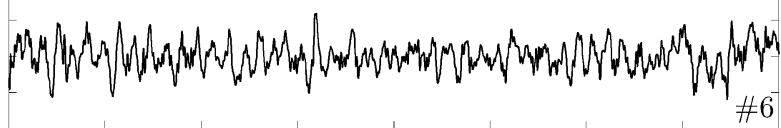

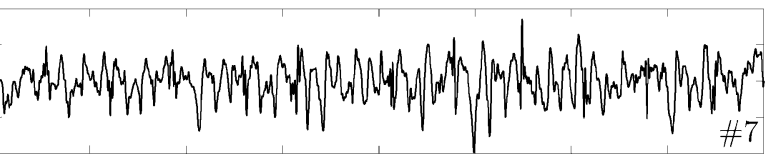

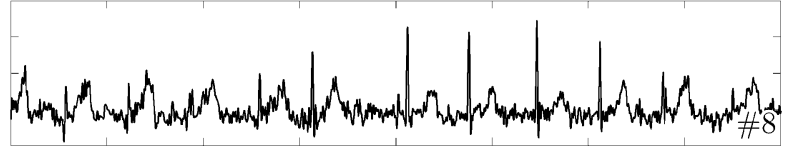

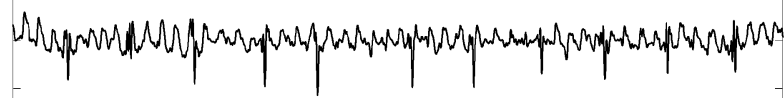

$\# 9$
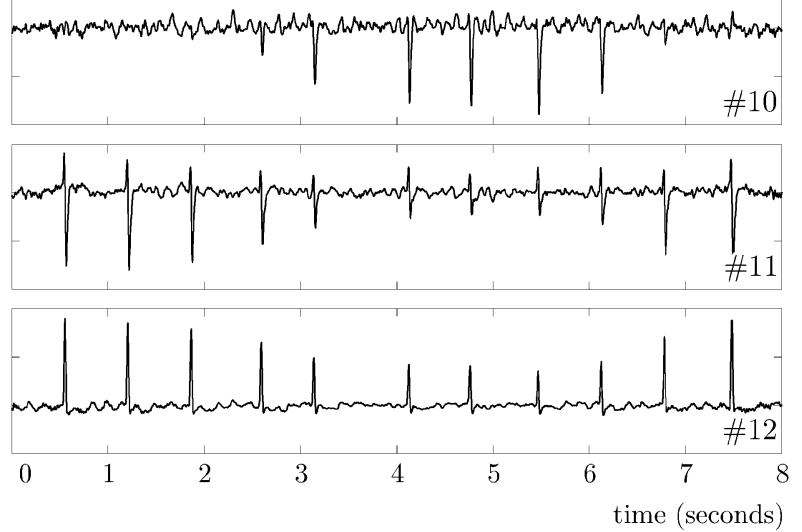

(b)

Fig. 2. Input and result of the ICA separation process. (a) A 12-lead ECG segment from a patient in AF. (b) Estimated sources obtained via ICA and reordered from lower to higher kurtosis value. The AA is contained in source \#1.

conventional quasi-static approximation [36]. That is, all currents and fields behave, at any instant, as if they were stationary and we can assume the fulfillment of the BSS instantaneous linear mixture model for (7).
The joint activity of the cardiac cells can be observed via the multilead ECG but it is evident that the mathematical operations that define the voltages for the 12-lead ECG are only linear combinations of the body surface potentials and, hence, do not af- 
fect at all the aforementioned instantaneous linear mixture BSS model. Then, the application of BSS-based methods on the standard ECG is completely justified and remarked with the duality between (7) and (1). As a consequence of the results from Sections II-C and II-D, the three most important requirements to apply the ICA-based BSS technique, namely, instantaneous linear mixtures, source independence, and non-Gaussianity, do indeed hold for the 12-lead ECG recordings of a patient with AF.

\section{METHODS}

By virtue of the previous discussion, the skin-electrode signal vector of the ECG can be identified with $\mathbf{x}(t)$ and complies with the generative BSS model in (1), where vector $\mathbf{s}(t)$ is composed of the independent sources of atrial and ventricular cardiac activity and other nuisance signals. The mixing matrix entries depend on the body geometry, tissue conductivity, and electrode position similarly as occurs in the BSS formulation of the fetal ECG extraction problem [17]. Consequently, the atrial contribution to the recordings can be recovered by extracting, via ICA, the sources of AA and the corresponding columns of the mixing matrix.

Before applying ICA, all signals were sampled (or upsampled from $500 \mathrm{~Hz}$ ) at $1 \mathrm{kHz}$ in order to improve frequency resolution when performing the spectral analysis and, at the same time, make use of the most standard sampling rate for ECG studies. The upsampling process consisted of low-pass FIR filtering the ECG segment and inserting new samples obtained via a nearest neighbor interpolation. After amplitude normalization the signals were preprocessed using a $50-\mathrm{Hz}$ notch filter to cancel out mains interference, followed by a band-pass filter with cut-off frequencies of 0.5 and $60 \mathrm{~Hz}$ to remove baseline wandering and reduce thermal noise [4].

The authors' own signal database comprised recordings from seven patients suffering from AF. All of the ECGs were composed of 12 leads and were $8 \mathrm{~s}$ in length. No dimensionality reduction was performed in the whitening process before ICA computation. The FastICA algorithm [37] was preferred to perform the BSS process, due to its fast convergence and robust performance, previously demonstrated in a variety of different applications [38]. In addition, FastICA can operate in a deflation mode, in which the independent components are estimated one by one. Hence, the algorithm can be stopped as soon as the AA sources have been extracted, with the consequent benefit in computational complexity.

After the application of ICA, the sub-Gaussian statistical character of AA as opposed to the super-Gaussian behavior of VA allows the identification of the estimated AA source using a kurtosis-based source reordering. This reordering process arranges first the sub-Gaussian sources, associated with AA, then the Gaussian ones, associated with noise and other artifacts, and finally the super-Gaussian sources, corresponding to VA. Therefore, according to the predicted statistical behavior of AA, the separated signals with lower kurtosis are considered to be the AA sources.

After the kurtosis-based reordering, in order to validate the AA identification, the power spectral density (PSD) was computed for all of the separated sources with sub-Gaussian kur-

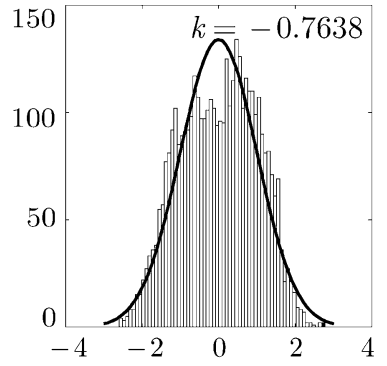

(a)

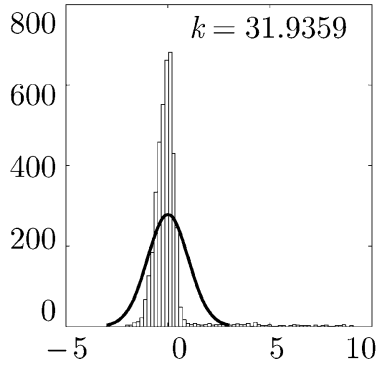

(b)
Fig. 3. Histogram of separated sources of Fig. 2, with superimposed Gaussian distribution. (a) Source \#1, associated with the AA signal estimate. (b) Source $\# 12$, associated with VA.

tosis $(k<0)$. The procedure consisted of obtaining the modified periodogram using the Welch-WOSA method [39] with a Hamming window of 4096 points length, a 50\% overlapping between adjacent windowed sections, and an 8192-point fast Fourier transform (FFT). Later, the spectral content above 20 $\mathrm{Hz}$ was discarded due to its low contribution. In this manner, it was possible to observe and compare the spectral content of the separated sources with the clinically accepted spectral content of AF [4], [11], [23], [40], [41].

\section{RESULTS}

After the ICA separation process, it was always possible to identify the AA source among the whole set of 12 separated sources. The identification was carried out following the aforementioned steps based on reordering the sources from lower to higher kurtosis, obtaining and analyzing the PSD of the sources with sub-Gaussian kurtosis, and, finally, visually inspecting the fibrillatory waves in the original ECG against the estimated AA source obtained by the ICA separation. Fig. 2(a) plots a 12-lead ECG with an AF episode. Observe the fibrillatory waves that can be clearly identified in several leads. It is generally accepted by the scientific community [1] that leads II, III, aVF, and especially V1 have the largest AA content, as can be seen in the figure.

The result of applying ICA to this AF episode and reordering the estimated sources as a function of its kurtosis generates the sources plot of Fig. 2(b), where source \#1 has the lowest kurtosis $(-0.76)$ and source \#12 has the largest one $(+31.93)$. Due to the kurtosis reordering, the first separated sources (\#1-3) have a more sub-Gaussian PDF and hence are the candidates to contain the AA, the central sources are associated with Gaussian noise and signal artifacts (\#4-7), and the last sources (\#8-12) mainly contain VA. Fig. 3 plots the histogram of sources \#1 and \#12 with a superimposed normal distribution with the same mean and variance. As can be observed, AA presents a sub-Gaussian character whereas VA exhibits a strong super-Gaussian behavior, as has been previously indicated in the AF BSS model of Section II-C.

Additionally, a spectral analysis is applied over the sources with sub-Gaussian kurtosis $(k<0)$ to determine the AA source. Fig. 4 plots the results of the PSD estimation for all the negative-kurtosis sources. As can be observed, source \#1 presents a typical spectral morphology of the AA from a patient with AF. 

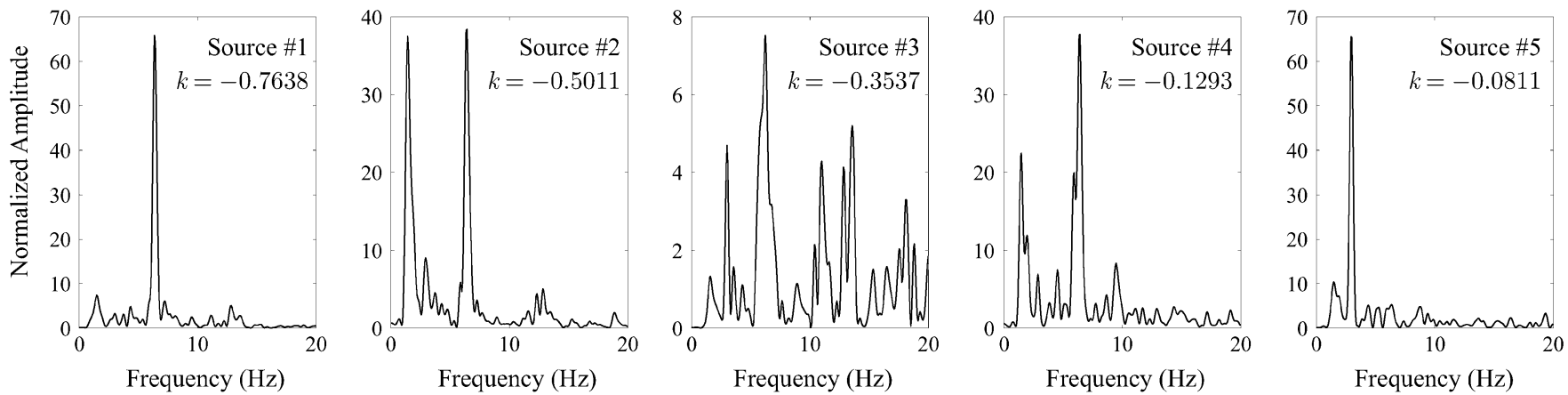

Fig. 4. Power spectral densities from several ICA-estimated sources of Fig. 2. After kurtosis-based reordering only five sources have sub-Gaussian kurtosis, and the one with lowest kurtosis (source \#1) presents a PSD typically associated with the AA in AF episodes.

TABLE I

COEFFicients OF THE Sixth COLumn OF THE Mixing Matrix SHOwing the Projection of SOURCE \#6 (CONSIDEREdTo BE THE AA) ONTO EACH ECG LEAD

\begin{tabular}{rrrrr}
\hline \hline Lead & Coeff. $a_{i, 6}$ & & Lead & Coeff. $a_{i, 6}$ \\
\cline { 1 - 2 } \cline { 5 - 5 } I & -0.0113 & & V1 & 0.0684 \\
II & -0.0137 & & V2 & 0.0067 \\
III & -0.0018 & & V3 & 0.0034 \\
aVR & 0.0130 & & V4 & -0.0030 \\
aVL & -0.0047 & & V5 & 0.0052 \\
aVF & -0.0078 & & V6 & -0.0065 \\
\hline \hline
\end{tabular}

The pattern of this type of episode is characterized by a very pronounced peak in frequencies from 5 to $8 \mathrm{~Hz}$, without harmonics and with insignificant amplitudes above $15 \mathrm{~Hz}$. In the case of source \#1, the main peak frequency is $f_{p}=6.34 \mathrm{~Hz}$. It can also be appreciated in Fig. 4 that the only separated source with similar spectral content is source \#5. However, the main peak frequency of this signal is $f_{p}=2.93 \mathrm{~Hz}$ and, thus, it cannot be considered as AA. This decision is further reinforced by its kurtosis value $\left(k_{5}=-0.081\right)$, which indicates a closer proximity to Gaussianity.

The application of the proposed BSS-based AA extraction procedure on the rest of the AF patient database consistently provided satisfactory results, as summarized in Fig. 5. These results correspond to patients \#2-\#7 (the results from patient \#1 are presented in Figs. 3, 4, and 6), where each row is associated with one patient. In the first column, lead V1 (in the bottom) can be observed from the 12-lead ECG in AF, along with the ICA-estimated AA for that episode (at the top) for visual comparison. The estimated AA has been scaled by the factor associated with its projection onto lead V1 (as will be shown later in Table II). The visual similarity between the estimated AA and the AA contained inside V1 is remarkable. The second column shows the estimated AA PSD along with the computed main peak frequency (atrial frequency). As can be appreciated, the spectral content associated with the estimated AA source is in agreement with the expected one associated with AF [4], [11], [23], [40], [41]. Finally, the third column shows the histogram of the AA estimated source for each patient with superimposed Gaussian distribution. In general, now we can say that the sub-Gaussian behavior of the estimated AA is not so far from Gaussianity. Hence, the kurtosis values (also indicated in the figure) are close to zero but are still negative.

\section{DISCUSSION}

After the use of the FastICA [37] approach over the ECG segments, additional ICA algorithms were applied to the signal database in order to compare results in the AA extraction process. The algorithms employed were AMUSE and JADE from ICALAB Toolbox [42] and HOEVD [10]. All cases yielded similar results. Note that the objective of this paper is to justify and show the use of ICA in solving the AA extraction problem in AF episodes rather than find out what ICA approach performs better in this concrete problem; this could be studied in future papers.

The direct visual identification of the AA source, after applying ICA to the ECG, is not always possible. The kurtosisbased source reordering, which takes advantage of the dissimilar statistical properties of AA and VA, has proven its ability to identify the AA component from the set of estimated source signals with the lower kurtosis values. The sub-Gaussian behavior of the estimated AA source in all patients analyzed has not been as pronounced as expected. All of the kurtosis values of the estimated AA sources have been negative, but not so far from zero (Gaussianity). Nevertheless, this result is not considered to be a problem for the separation of AA from Gaussian noise. Though ICA can separate at most one Gaussian source and, hence, Gaussian noise could not be separated from nearGaussian AAs, the noise power in the ECG is much smaller than AA, as demonstrated in the results. Moreover, it could be possible to separate the AA from Gaussian noise via their very dissimilar spectral contents. In a second step, the AA identification process has been completed with the spectral analysis of the sub-Gaussian sources. The combination of these two steps constitutes a robust AA identification method from the BSS results.

The AA estimates obtained by BSS from these ECGs in AF were considered by cardiologists as very approximate to the real atrial waveforms contained in the episode. This outcome is illustrated in Fig. 6, which shows (in the top) the atrial source \#1 of Fig. 2 estimated via BSS scaled by the factor 0.0684 , which corresponds to the projection of the estimated AA onto lead V1. V1 is usually accepted as the lead with the largest AA content and is shown in the bottom of Fig. 6 for visual comparison. Shown in the middle of Fig. 6 is the AA estimation result obtained when PCA is applied over the same ECG. As has been pointed out in 

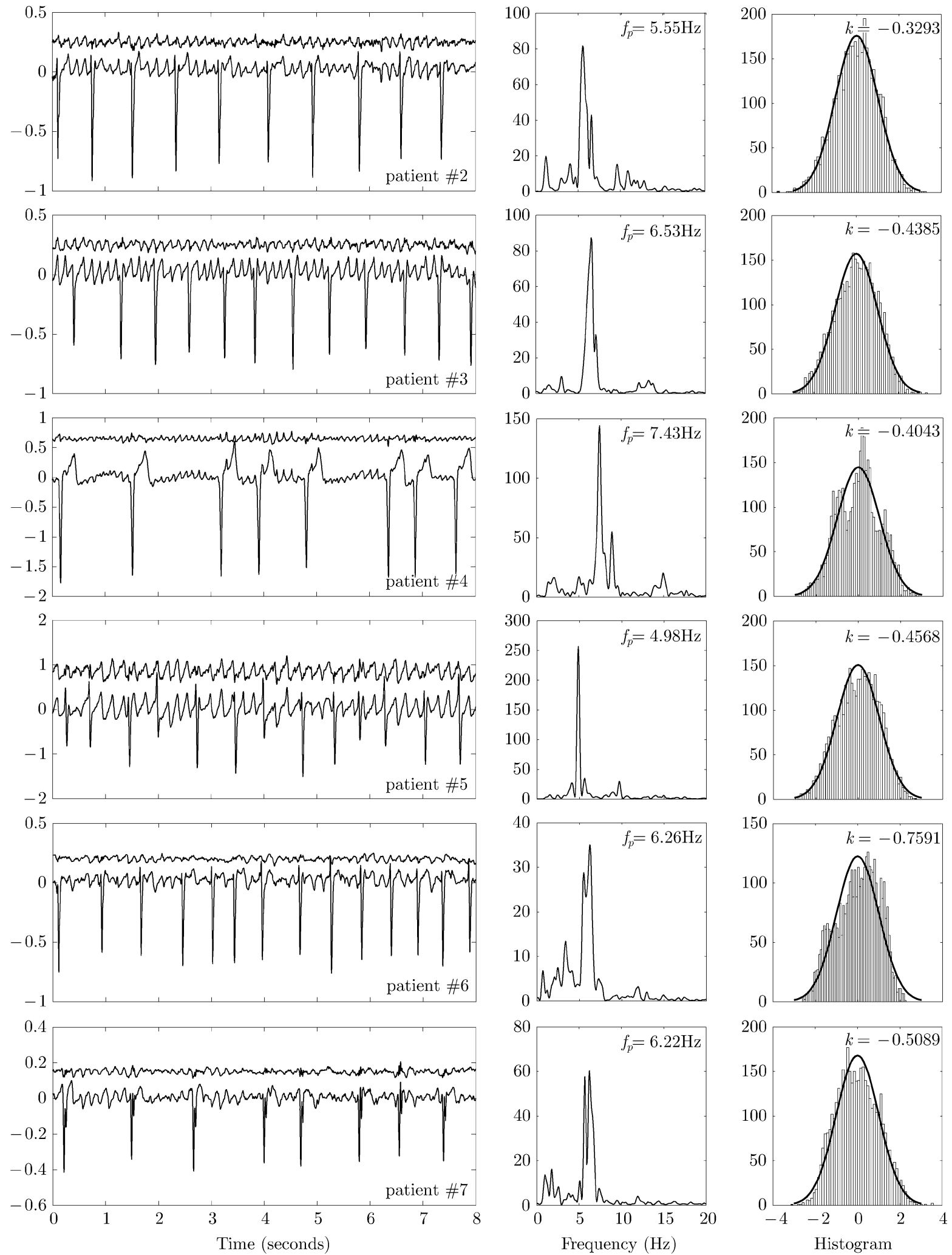

Fig. 5. AA extraction results from patients \#2-\#7 (one patient per row). The first column shows the estimated AA source (top) and lead V1 (bottom). The second column shows the PSD computed for the estimated AA along with the atrial frequency. The third column shows the histogram of the estimated AA with superimposed Gaussian distribution (of the same mean and variance) and its kurtosis value.

previous sections, the VA cancellation in this case is not as good as that in ICA. This can be especially observed in the R-peaks. Similar results have been reported in [41].

Before applying the kurtosis-based reordering to the estimated sources (as shown in Fig. 2), the AA obtained by the
ICA separation process was present in source \#6. Hence, the sixth column of the estimated mixing matrix $\mathbf{A}$ indicates how the associated source is projected onto the observations. Table I shows the projection of the AA estimated source (\#1 in Figs. 2 and 4) to each observation. Clearly, lead V1 has the largest 
TABLE II

Projection COEFFICIENTS of THE Estimated AA SOURCES ONTO THE ECG LEADS OF PATIENTS \#2-\#7

\begin{tabular}{|c|c|c|c|c|c|c|c|c|c|c|c|c|}
\hline Leads & I & II & III & aVR & aVL & aVF & V1 & V2 & V3 & V4 & V5 & V6 \\
\hline Patient \#2 & -0.0103 & 0.0052 & 0.0151 & 0.0029 & -0.0131 & 0.0099 & 0.0254 & 0.0054 & -0.0048 & 0.0200 & 0.0205 & 0.0089 \\
\hline Patient \#3 & -0.0067 & -0.0207 & -0.0126 & 0.0140 & 0.0025 & -0.0166 & -0.0307 & -0.0273 & -0.0256 & -0.0206 & -0.0164 & -0.0159 \\
\hline Patient \#4 & 0.0094 & -0.0112 & -0.0200 & 0.0015 & 0.0147 & -0.0153 & 0.0365 & 0.0251 & 0.0243 & 0.0268 & 0.0204 & 0.0236 \\
\hline Patient \#5 & -0.0040 & -0.0074 & -0.0031 & 0.0051 & -0.0005 & -0.0051 & 0.0379 & 0.0194 & 0.0160 & 0.0198 & 0.0103 & 0.0189 \\
\hline Patient \#6 & 0.0464 & -0.1178 & -0.1288 & 0.0178 & 0.0903 & 0.0631 & -0.6715 & -0.3314 & -0.2515 & -0.0159 & -0.1033 & 0.0485 \\
\hline Patient \#7 & 0.0084 & 0.0223 & 0.0143 & -0.0155 & -0.0029 & 0.0185 & -0.0313 & -0.0249 & -0.0172 & -0.0280 & -0.0098 & -0.0217 \\
\hline
\end{tabular}

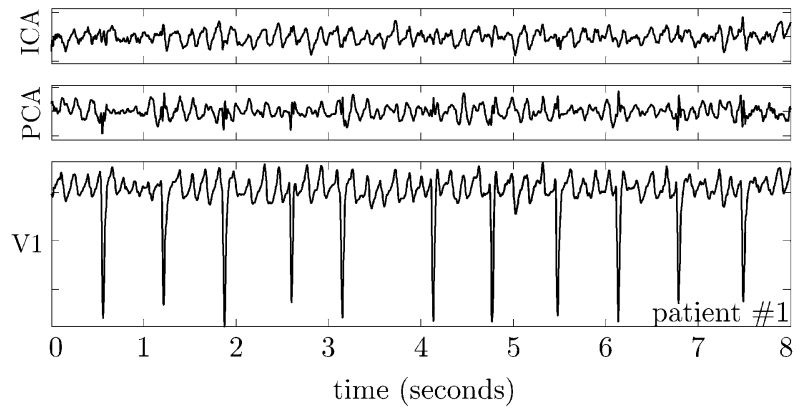

Fig. 6. Visual comparison of the reconstructed AA contribution to lead V1. At the top, separated source \#1 of Fig. 2, associated with the AA signal estimate using ICA, is scaled by coefficient 0.0684 which corresponds to the projection of this source on the observation lead V1. In the middle is shown the result of the same process using PCA. The bottom shows lead V1 of the 12-lead ECG in Fig. 2.

contribution from the estimated AA source. This result, which is in close agreement with clinical experience, is an additional indication of the AA extraction quality. In the cases where the absolute amplitude of the extracted AA using BSS could be of clinical interest, it is possible to reconstruct it back to each ECG lead using the aforementioned column coefficients.

Note that we are dealing with an inverse problem, where the true sources are not accessible (noninvasively, at least), and hence the difficulty in evaluating the success of the AA source separation. One is left with estimating the AA contribution to the ECG leads typically containing the largest AA and making a visual comparison of the corresponding fibrillatory waves. Despite the large visual similarity between the fibrillatory waves of the estimated AA source and the AA contained in lead V1 (see Fig. 6), it must be said that this kind of direct visual comparison, strictly speaking, only has to be considered in an illustrative way, because the obtained AA source via BSS combines AA information from all of the ECG leads and not only from V1. Nevertheless, the only way to corroborate if the AA source separation has been satisfactory is to compare it with those ECG leads containing the largest atrial activity. This is a typical consequence of the BSS-based methods where the real sources are latent variables that cannot be directly observed.

Finally, Table II shows the projection coefficients of the estimated AA source corresponding to patients \#2-\#7. As can be seen (similarly as in Table I), lead V1 contains the largest AA contribution. Nevertheless, it can also be observed that the estimated AA is spread over all of the ECG leads (for a given patient, all of the projection coefficients are nonzero). This observation demonstrates the presence of AA in all of the leads, and, at the same time, the power of this ICA-based AA extraction technique, capable of taking into account the atrial contribution in every lead to generate a unified signal estimate condensing the AA information. The authors also have verified that similarly good results can be obtained in other supraventricular arrhythmias, like atrial flutter [23], and hope that this new methodology will also work in cardiac pathologies where atrial and ventricular activities are unsynchronized or decoupled, like in the AV-block.

Nevertheless, note that BSS techniques are based on statistical analysis of the data, and hence its results will not be satisfactory if the data given to the algorithm are incorrect. Therefore, it will only be possible to derive the spatial filters associated with the mixing matrix entries and the independent sources from the ECG, when the physical sources associated with the heart's bioelectrical activity are spatially stationary in time and the total number of these sources is less than the number of observations (ECG leads), as indicated in [43]. Strictly speaking, movements of the heart, such as contraction of the atria and ventricles, could violate the ICA assumption of spatial stationarity of the physical sources but, in general, the authors consider that these possible variations do not significantly affect the BSS instantaneous linear mixing model for AF episodes. This consideration is reinforced by the fact that results providing the estimation of the main atrial frequency of AA using this ICA-based BSS technique are the same as those obtained through the application of other accepted AA extraction techniques, as proved in [41].

\section{CONCLUSION}

This paper has shown that the noninvasive extraction of AA in AF episodes recorded from the surface ECG can be effectively carried out by HOS-based BSS techniques for instantaneous linear mixtures. The applicability of this type of technique in this biomedical problem has been discussed in connection with its three main assumptions. First, in atrial arrhythmia episodes, the cardioelectric sources generating AA and VA can be regarded as statistically independent. Second, both activities present a non-Gaussian character. Finally, AA and VA are manifested on the body surface as an instantaneous linear mixture of the cardiac sources, in which the unknown mixture coefficients depend on the ECG electrode position and the conductivity of the body tissues. The justification of these key assumptions makes feasible the application of HOS-based BSS, and this contribution has indeed demonstrated its usefulness to solve the AA extraction problem. Traditional techniques obtain as many AA signals as leads processed by the cancellation algorithm; in contrast, the BSS-based method estimates a single signal which is able to reconstruct the complete AA present in every ECG lead. On the other hand, the BSS approach can be considered 
as an alternative procedure for (indirect) QRST cancellation in atrial arrhythmia analysis.

The positive results reported in this paper mean the advent of novel noninvasive techniques for AF analysis and are the first step in the development and future improvement of new diagnostic strategies, pathology prediction methodologies, and aid tools based on AA-wave analysis in the management of patients with AF. In fact, most of the actual diagnosis and management of patients with AF are judged on the basis of clinical symptoms and ECG recordings. Therefore, the development and availability of suitable techniques allowing the knowledge of AF patterns (paroxysmal, persistent, or permanent) and aiding in the decision making about restoration and maintenance of sinus rhythm or control of the ventricular rate may be a tool of fundamental importance for the treatment of AF, a commonly encountered arrhythmia in permanent expansion.

\section{ACKNOWLEDGMENT}

The authors would like to thank cardiologists R. Ruiz Granell, S. Morell, F. J. Chorro, and R. García Civera, from the Electrophysiology Laboratory of the Universitary Clinical Hospital of Valencia, Spain, for their clinical advice and kind help in obtaining the signals.

\section{REFERENCES}

[1] V. Fuster, L. E. Ryden, R. W. Asinger, D. S. Cannom, H. J. Crijns, R. L. Frye, J. L. Halperin, G. N. Kay, W. W. Klein, S. Levy, R. L. McNamara, E. N. Prystowsky, L. S. Wann, and D. G. Wyse, "ACC/AHA/ESC guidelines for the management of patients with atrial fibrillation: a report of the American College of Cardiology/American Heart Association Task Force on Practice Guidelines and the European Society of Cardiology Committee for Practice Guidelines and Policy Conferences (Committee to Develop Guidelines for the Management of Patients With Atrial Fibrillation)," J. Amer. Coll. Cardiol., vol. 38, pp. 1266 i-1266 lxx, 2001.

[2] S. Levy et al., "Atrial fibrillation: Current knowledge and recommendations for management," Eur. Heart J., vol. 19, no. 9, pp. 1294-1320, 1998.

[3] R. H. Falk, "Medical progress: Atrial fibrillation," New Eng. J. Med., vol. 344, no. 14, pp. 1067-1078, 2001.

[4] A. Bollmann, N. K. Kanuru, K. K. McTeague, P. F. Walter, D. B. DeLurgio, and J. J. Langberg, "Frequency analysis of human atrial fibrillation using the surface electrocardiogram and its response to ibutilide," Amer. J. Cardiol., vol. 81, no. 12, pp. 1439-1445, 1998.

[5] N. V. Thakor and Y. S. Zhu, "Applications of adaptive filtering to ecganalysis: Noise cancellation and arrhytmia detection," IEEE Trans. Biomed. Eng., vol. 38, pp. 785-794, Aug. 1991.

[6] S. Shkurovich, A. V. Sahakian, and S. Swiryn, "Detection of atrial activity from high-voltage leads of implantable ventricular defibrillators using a cancellation technique," IEEE Trans. Biomed. Eng., vol. 45, pp. 229-234, Feb. 1998.

[7] M. Stridh and L. Sörnmo, "Spatiotemporal QRST cancellation techniques for analysis of atrial fibrillation," IEEE Trans. Biomed. Eng., vol. 48, pp. 105-111, Jan. 2001.

[8] C. Vasquez, A. Hernandez, F. Mora, G. Carrault, and G. Passariello, "Atrial activity enhancement by wiener filtering using an artificial neural network," IEEE Trans. Biomed. Eng., vol. 48, pp. 940-944, Aug. 2001

[9] C. Sanchez, J. Millet, J. J. Rieta, J. Rodenas, F. Castells, R. Ruiz, and V. Ruiz, "Packet wavelet decomposition: An approach for atrial activity extraction," IEEE Comput. Cardiol., vol. 29, pp. 33-36, Sept. 2001.

[10] V. Zarzoso and A. K. Nandi, "Blind source separation," in Blind Estimation Using Higher-Order Statistics, A. K. Nandi, Ed. Boston, MA: Kluwer, 1999, pp. 167-252.

[11] P. Langley, J. P. Bourke, and A. Murray, "Frequency analysis of atrial fibrillation," IEEE Comput. Cardiol., vol. 27, pp. 65-68, Sept. 2000.

[12] L. Faes, G. Nollo, M. Kirchner, E. Olivetti, F. Gaita, R. Riccardi, and R. Antolini, "Principal component analysis and cluster analysis for measuring the local organization of human atrial fibrillation," Med. Biol. Eng. Comput., vol. 39, pp. 656-663, 2001.
[13] J. J. Rieta, V. Zarzoso, J. Millet, R. Garcia, and R. Ruiz, "Atrial activity extraction based on blind source separation as an alternative to QRST cancellation for atrial fibrillation analysis," IEEE Comput. Cardiol., vol. 27, pp. 69-72, Sept. 2000.

[14] J. J. Rieta, F. Castells, C. Sanchez, and J. Igual, "ICA applied to atrial fibrillation analysis," in Proc. Int. Conf. Independent Component Analysis and Blind Signal Separation (ICA), vol. 4, Nara, Japan, 2003, pp. 59-64.

[15] R. Vigario, J. Sarela, V. Jousmaki, M. Hamalainen, and E. Oja, "Independent component approach to the analysis of EEG and MEG recordings," IEEE Trans. Biomed. Eng., vol. 47, pp. 589-593, May 2000.

[16] T. P. Jung, S. Makeig, C. Humphries, T. W. Lee, M. J. McKeown, V. Iragui, and T. J. Sejnowski, "Removing electroencephalographic artifacts by blind source separation," Psychophysiol., vol. 37, no. 2, pp. 163-178, 2000.

[17] V. Zarzoso and A. K. Nandi, "Noninvasive fetal electrocardiogram extraction: Blind separation versus adaptive noise cancellation," IEEE Trans. Biomed. Eng., vol. 48, pp. 12-18, Jan. 2001.

[18] J. O. Wisbeck, A. K. Barros, and R. C. Ojeda, "Application of ica in the separation of breathing artifacts in ECG signals," in Proc. 5th Int. Conf. Neural Information Processing (ICONIP'98), vol. 1, pp. 211-214.

[19] A. K. Barros, A. Mansour, and N. Ohnishi, "Adaptive blind elimination of artifacts in ECG signals," in Proc. Int. Workshop Independence \& Artificial Neural Networks (I\&ANN'98), pp. 1380-1386.

[20] T. Stamkopoulos, K. Diamantaras, N. Maglaveras, and M. Strintzis, "ECG analysis using nonlinear PCA neural networks for ischemia detection," IEEE Trans. Signal Processing, vol. 46, pp. 3058-3067, Nov. 1998.

[21] L. Biel, O. Pettersson, L. Philipson, and P. Wide, "ECG analysis: A new approach in human identification," IEEE Trans. Instrum. Meas., vol. 50, pp. 808-812, June 2001.

[22] M. I. Owis, A. B. M. Youssef, and Y. M. Kadah, "Characterization of electrocardiogram signals based on blind source separation," Med. Biol. Eng. Comput., vol. 40, no. 5, pp. 557-564, 2002.

[23] J. J. Rieta, J. Millet, V. Zarzoso, F. Castells, C. Sanchez, R. Garcia, and S. Morell, "Atrial fibrillation, atrial flutter and normal sinus rhythm discrimination by means of blind source separation and spectral parameters extraction," IEEE Comput. Cardiol., vol. 29, pp. 25-28, 2002.

[24] J. F. Cardoso, "Blind signal separation: Statistical principles," Proc. IEEE, vol. 86, pp. 2009-2025, Oct. 1998

[25] P. Comon, "Independent component analysis, a new concept?," Signal Processing, vol. 36, no. 3, pp. 287-314, 1994.

[26] J. Vanderschoot, D. Callaerts, W. Sansen, J. Vandewalle, G. Vantrappen, and J. Janssens, "Two methods for optimal MECG elimination and FECG detection from skin electrode signals," IEEE Trans. Biomed. Eng., vol. 34, pp. 233-243, Mar. 1987.

[27] D. Callaerts, B. Demoor, J. Vandewalle, W. Sansen, G. Vantrappen, and J. Janssens, "Comparison of SVD methods to extract the fetal electrocardiogram from cutaneous electrode signals," Med. Biol. Eng. Comput., vol. 28, no. 3, pp. 217-224, 1990.

[28] A. Hyvarinen, J. Karhunen, and E. Oja, Independent Component Analysis. New York: Wiley, 2001.

[29] J. Malmivuo and R. Plonsey, Bioelectromagnetism: Principles and Applications of Bioelectric and Biomagnetic Fields. Oxford, U.K.: Oxford Univ. Press, 1995.

[30] G. K. Moe, "On multiple wavelet hypothesis of atrial fibrillation," Archives Internationales de Pharmacodynamie et de Therapie, vol. 140, no. 1-2, pp. 183-188, 1962.

[31] R. M. Gulrajani, "The forward and inverse problems of electrocardiography," IEEE Eng. Med. Biol. Mag., vol. 17, pp. 84-101, May 1998.

[32] R. C. Barr, T. C. Pilkington, J. P. Boineau, and M. S. Spach, "Determining surface potentials from current dipoles, with application to electrocardiography," IEEE Trans. Biomed. Eng., vol. BME-13, pp. 88-92, 1966.

[33] R. C. Barr, M. Ramsey, and M. S. Spach, "Relating epicardial to body surface potential distribution by means of transfer coefficients based on geometry measurements," IEEE Trans. Biomed. Eng., vol. BME-24, pp. 1-11, Jan. 1977.

[34] R. N. Klepfer, C. R. Johnson, and R. S. MacLeod, "The effects of inhomogeneities and anisotropies on electrocardiographic fields: A 3-d finite-element study," IEEE Trans. Biomed. Eng., vol. 44, pp. 706-719, Aug. 1997.

[35] S. J. Walker and D. Kilpatrick, "Forward and inverse electrocardiographic calculations using resistor network models of the human torso," Circ. Res., vol. 61, no. 4, pp. 504-513, 1987.

[36] R. Plonsey and D. B. Heppner, "Considerations of quasistationarity in electrophysiological systems," Bull. Math. Biophys., vol. 29, no. 4, pp. 657-664, 1967. 
[37] The Fastica Package for Matlab, J. Hurri, J. Gavert, J. Sarela, and A. Hyvarinen. (1998). [Online]. Available: http://www.cis.hut.fi/ projects/ica/fastica

[38] A. Hyvarinen, "Fast and robust fixed-point algorithms for independent component analysis,", IEEE Trans. Neural Networks, vol. 10, pp. 626-634, May 1999.

[39] P. D. Welch, "Use of fast fourier transform for estimation of power spectra: A method based on time averaging over short modified periodograms," IEEE Trans. Audio Electroacoust., vol. AE-15, no. 2, pp. 70-73, 1967.

[40] M. Stridh, L. Sörnmo, C. J. Meurling, and S. B. Olsson, "Characterization of atrial fibrillation using the surface ECG: Time-dependent spectral properties," IEEE Trans. Biomed. Eng., vol. 48, pp. 19-27, Jan. 2001.

[41] P. Langley, M. Stridh, J. J. Rieta, L. Sörnmo, J. Millet, and A. Murray, "Comparison of atrial rhythm extraction techniques for the detection of the main atrial frequency from the 12-lead ECG in atrial fibrillation," IEEE Comput. Cardiol., vol. 29, pp. 29-32, Sept. 2002.

[42] Icalab Toolboxes, A. Cichocki, S. Amari, K. Siwek, T. Tanaka, and T. Rutkowski. (2003). [Online]. Available: http://www.bsp.brain. riken.go.jp/ICALAB

[43] T. P. Jung, S. Makeig, T. W. Lee, M. J. McKeown, G. Brown, A. J. Bell, and T. J. Sejnowski, "Independent component analysis of biomedical signals," in Proc. Int. Conf. Independent Component Analysis and Blind Signal Separation (ICA), vol. 2, 2000, pp. 633-644.

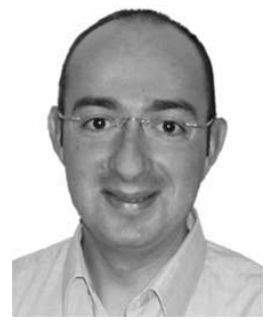

José Joaquín Rieta received the M. Eng. degree in image and sound from the Polytechnic University of Madrid, Madrid, Spain, in 1991, the M. Sc. degree in telecommunications from the Polytechnic University of Valencia, Valencia, Spain, in 1996 and the Ph.D. degree in Biomedical Signal Processing in 2003 in the same university.

Since 1994, he has been a Lecturer with the Electronic Engineering Department in the Valencia University of Technology, developing his teaching responsibilities at the Gandía Higher School of Technology. As lecturer he has taught several subjects related to electronic and biomedical instrumentation, analog systems, data conversion systems and control engineering, and has been the author of several docent publications in these areas. He belongs to the Bioengineering, Electronic and Telemedicine (BeT) research group where is the responsible for the Advanced Signal Processing research line. His research interests include statistical signal and array processing applied to biomedical signals, specially focused in cardiac signals, blind signal separation techniques, and the develop of clinical applications to study and characterize the atrial activity inside the challenging problem of supraventricular arrhythmias.

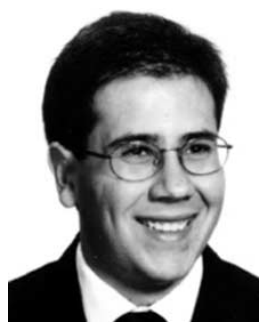

Francisco Castells was born in Spain in 1976. He received the M.Eng. degree in telecommunications engineering from the Universidad Politécnica de Valencia (UPV), Valencia, Spain, in 2000, where he is currently working toward the Ph.D. degree.

After working in the telecommunications manufacturing industry for Alcatel SEL AG in Germany (2000-2001), he started his PhD. studies at the UPV in 2002. He is currently an Associate Lecturer with the Department of Electronic Engineering, UPV, where he is also a member of the Bioengineering, Electronic and Telemedicine $(\mathrm{BeT})$ research group. His research interests lie in the area of biomedical signal processing, with special emphasis on the application of blind signal processing techniques to atrial fibrillation analysis.

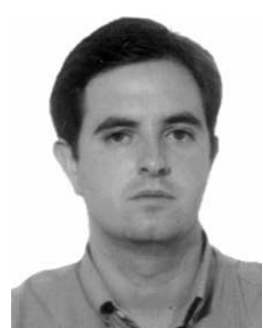

César Sánchez was born in Albacete, Spain, in 1973. He received the M.Eng. degree in telecommunications engineering from the Universidad Politécnica de Madrid, Madrid, Spain, in 1998 and he is currently working toward the Ph.D. degree at the Universidad Politécnica de Valencia (UPV), Valencia, Spain, in 2000.

He is currently a Lecturer with the Universidad de Castilla_La Mancha (UCLM), Spain, and works as an external member of the Bioengineering, Electronic and Telemedicine $(\mathrm{BeT})$ research group of the UPV. His research interests are centered on the application of advanced signal processing techiques such as the wavelet transform to cardiac signals.

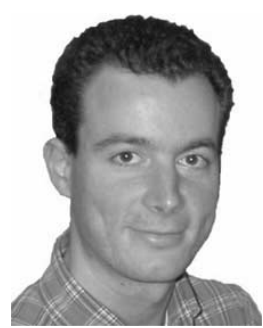

Vicente Zarzoso (S'94-A'99) was born in Valencia, Spain, on September 12, 1973. He received the degree in telecommunications engineering with the highest distinction (Premio Extraordinario de Terminación de Estudios) from the Universidad Politécnica de Valencia, Valencia, Spain, in 1996, and the Ph.D. degree from the University of Liverpool, Liverpool, U.K., in 1999.

He was awarded a scholarship by the University of Strathclyde, Glasgow, U.K., to study in the Department of Electronic and Electrical Engineering toward his Ph.D. degree, which was also funded in part by the Defence Evaluation and Research Agency (DERA) of the U.K. Since September 2000, he is in receipt of a Post-doctoral Research Fellowship awarded by the Royal Academy of Engineering of the U.K. He is currently on leave at the Laboratoire de Informatique, Signaux et Systèmes (I3S), Université de Nice, Sophia-Antipolis, France. His research interests include blind statistical signal and array processing and its application to biomedical problems and communications.

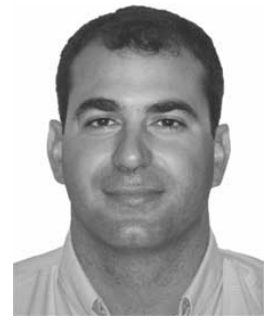

José Millet was born in Valencia, Spain, in 1968. He received the M.S. degree in physics from the University of Valencia (UV), Valencia, Spain, in 1991 and the $\mathrm{Ph} . \mathrm{D}$. degree in electrical and electronic engineering from the Universidad Politécnica de Valencia (UPV), Valencia, Spain, in 1997.

$\mathrm{He}$ is currently an Associate Professor of Electronics and Biomedical Signal Processing with the Electronic Engineering Department, UPV. From 1991 to 1999 , he worked as Assistant Professor in the same department. Since 1997, he has been the coordinator of the Biomedical Engineering branch within the Bioengineering, Electronic and Telemedicine (BeT) research group of UPV. His professional research interests are in biomedical signal processing, biomedical signal acquisition and instrumentation, implantable devices for treatment of cardiac arrhythmias, and cardiac MRI. 